\title{
Is the baryon acoustic oscillation peak a cosmological standard ruler?
}

\author{
Boudewijn F. Roukema ${ }^{1,2}$, Thomas Buchert ${ }^{2,3}$, Hirokazu Fujii ${ }^{4}$, Jan J. Ostrowski ${ }^{1,2}$ \\ ${ }^{1}$ Toruń Centre for Astronomy, Faculty of Physics, Astronomy and Informatics, Grudziadzka 5, Nicolaus Copernicus University, ul. Gagarina 11, \\ 87-100 Toruń, Poland \\ ${ }^{2}$ Université de Lyon, Observatoire de Lyon, Centre de Recherche Astrophysique de Lyon, CNRS UMR 5574: Université Lyon 1 and École Normale \\ Supérieure de Lyon, 9 avenue Charles André, F-69230 Saint-Genis-Laval, France* \\ ${ }^{3}$ Departamento de Astronomía, Universidad de Chile, Camino del Observatorio 1515, Santiago, Chile \\ ${ }^{4}$ Institute of Astronomy, University of Tokyo, 2-21-1 Osawa, Mitaka, Tokyo 181-0015, Japan
}

Le 25 septembre 2015

\begin{abstract}
In the standard model of cosmology, the Universe is static in comoving coordinates; expansion occurs homogeneously and is represented by a global scale factor. The baryon acoustic oscillation (BAO) peak location is a statistical tracer that represents, in the standard model, a fixed comoving-length standard ruler. Recent gravitational collapse should modify the metric, rendering the effective scale factor, and thus the BAO standard ruler, spatially inhomogeneous. Using the Sloan Digital Sky Survey, we show to high significance $(P<0.001)$ that the spatial compression of the BAO peak location increases as the spatial paths' overlap with superclusters increases. Detailed observational and theoretical calibration of this BAO peak location environment dependence will be needed when interpreting the next decade's cosmological surveys.
\end{abstract}

Key words: Cosmology: observations - cosmological parameters - distance scale - largescale structure of Universe - dark energy

\section{INTRODUCTION}

The choice of a spacetime coordinate system for the Universe that enables the expansion to be represented via a global scale factor $a$ as a function of just one coordinate (cosmological time $t$, Lemaitre e.g. 1927) is extremely convenient. On large enough comoving length scales, statistical spatial patterns are fixed in this coordinate system. Thus, the theory of primordial density fluctuations leads to that of baryon acoustic oscillations (Eisenstein \& Hu 1998; BAOs). The BAO peak in the two-point spatial auto-correlation function $\xi$ was clearly detected in the Sloan Digital Sky Survey (Eisenstein et al. 2005) and the Two-Degree Field Galaxy Redshift Survey (Cole et al. 2005); earlier surveys may have detected this too, Einasto et al. 1997). BAOs now constitute one of the most important tools for making cosmological geometry measurements, especially for upcoming observational projects such as the space mission Euclid (Refregier et al. 2010) and the ground-based instruments DESI (Levi et al. 2013), 4MOST (de Jong et al. 2012), and the LSST (Tyson et al. 2003). The BAO peak location of about $105 h^{-1} \mathrm{Mpc}$ (where $h$ is the Hubble constant $H_{0}$ expressed in units of $100 \mathrm{~km} / \mathrm{s} / \mathrm{Mpc}$ ) is commonly expected to be a large enough comoving length scale for it to provide a fixed comoving ruler in the real Universe.

* BFR: during visiting lectureship; JJO: during long-term visit.
However, the validity of the BAO peak location as a standard ruler depends on the validity of the assumed cosmological metric (differential rule for measuring lengths) in the context of the real Universe, which is lumpy (cf. the "fitting problem", Ellis \& Stoeger 1987). Scalar averaging is a general-relativistic formalism that extends beyond the standard cosmological model, by allowing a spatial section of the Universe at a given time to have an inhomogeneous metric and calculating background-free volumeweighted averages of scalar variables (Buchert 2001, 2008). The univariate scale factor $a(t)$ is replaced by an effective, environmentdependent volume-based scale factor $a_{\mathcal{D}}(t) \propto V_{\mathcal{D}}^{1 / 3}$, dependent on both the choice of compact spatial domain $\mathcal{D}$ of volume $V_{\mathcal{D}}$ and on time. Without this extension, the cosmological, comoving metric is forced (by definition) to be rigid in comoving coordinates, i.e. inhomogeneities in the matter distribution are not allowed to "tell comoving space how to curve" (e.g. Buchert \& Carfora 2008). Applying scalar averaging to an observationally standard power spectrum that statistically represents density fluctuations at an early epoch implies that even for spatial domains as large as the BAO peak length scale, the environment dependence of the scale factor should be observationally detectable, i.e. $a_{\mathcal{M}}<a_{\mathcal{E}}$ is expected, where $\mathcal{M}$ ("Massive") and $\mathcal{E}$ ("Empty") represent overdense and underdense spatial regions, respectively. For example, adopting $1 \sigma$ initially overdense $(\mathcal{M})$ and underdense $(\mathcal{E})$ fluctuations in a spherical domain of diameter $\approx 105 h^{-1} \mathrm{Mpc}$ and using equations (2), 

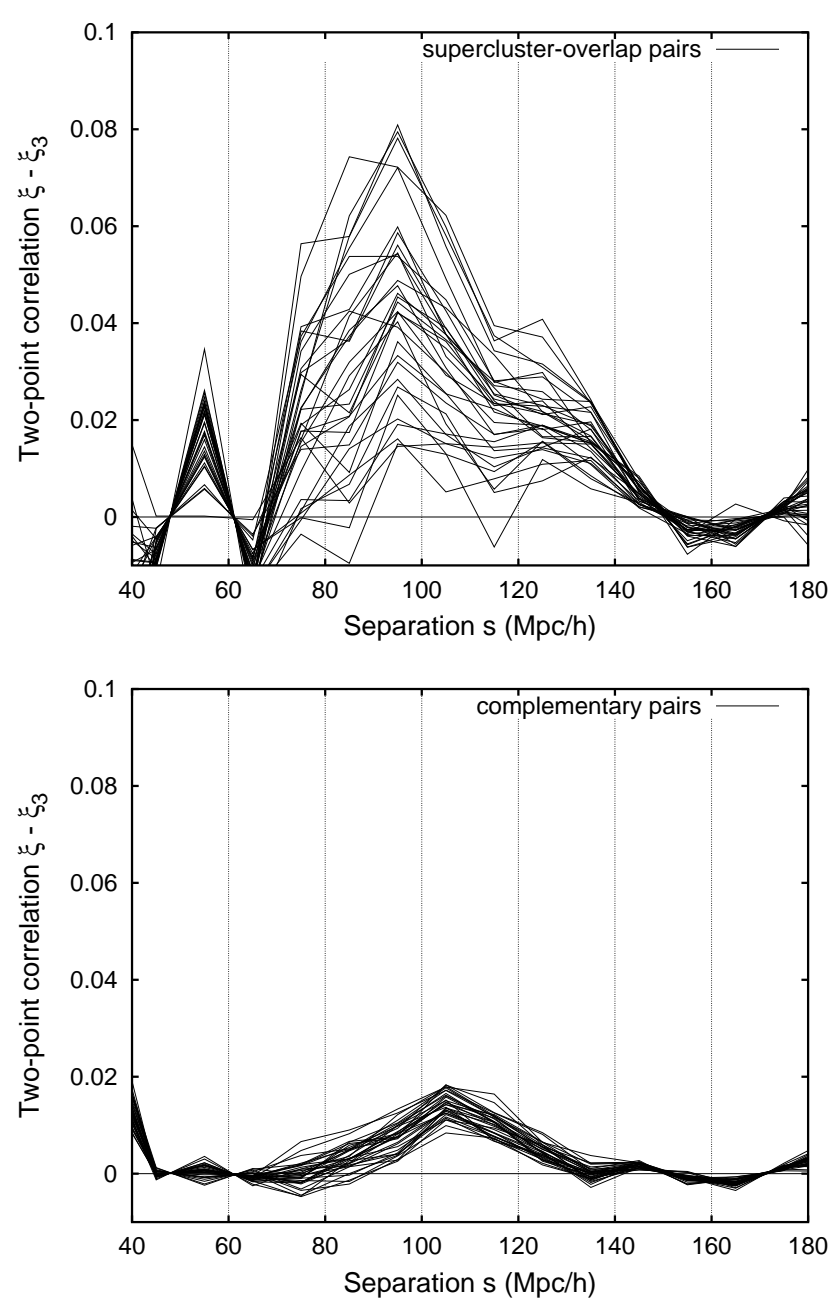

Figure 1. Compression of the baryon acoustic oscillation peak. Upper panel: BAO peak (cubic-subtracted correlation function) for pairs of luminous red galaxies whose paths either overlap with superclusters by $\omega \geq \omega_{\min }=60 h^{-1} \mathrm{Mpc}$ or are entirely contained within the superclusters. The overlap $\omega$ is the chord length defined (Roukema et al. 2015, Sect. 2.3, Fig. 1) by the intersection of the path joining two LRGs and the supercluster modelled as a sphere. Individual curves represent 32 bootstrap resamplings of the supercluster catalogue (235 objects) and the "random" galaxies $(484,352$ selected from 1,521,736). The real galaxies $(30,272)$ are not resampled. Most of the curves peak sharply at $95 h^{-1} \mathrm{Mpc}$; a few peak at $85 h^{-1} \mathrm{Mpc}$. The high amplitude (in comparison with the lower panel) is consistent with biasing that modifies the amplitude of $\xi$. Lower panel: BAO peak for the complementary subset of galaxy pairs. The peak occurs at the standard value of about $105 h^{-1} \mathrm{Mpc}$.

(13), (32), (50), and (54) of Buchert, Nayet \& Wiegand (2013) to integrate the Raychaudhuri equation (9) of the same paper gives a relativistic Zel'dovich approximation estimate of

$a_{\mathcal{M}} / a_{\mathcal{E}} \approx 0.91$.

In other words, in the scalar averaging approach, one way in which matter inhomogeneities are expected to affect the large-scale geometry and dynamics is to shrink the curved-space volume of overdensities on the BAO scale by about $1-\left(a_{\mathcal{M}} / a_{\mathcal{E}}\right)^{3} \approx 24 \%$, leading to an expected shift in the BAO peak location to a lower scale by somewhat below or above $9 \%$ for galaxy pairs that weakly or strongly, respectively, overlap with typical BAO-scale overdensities.

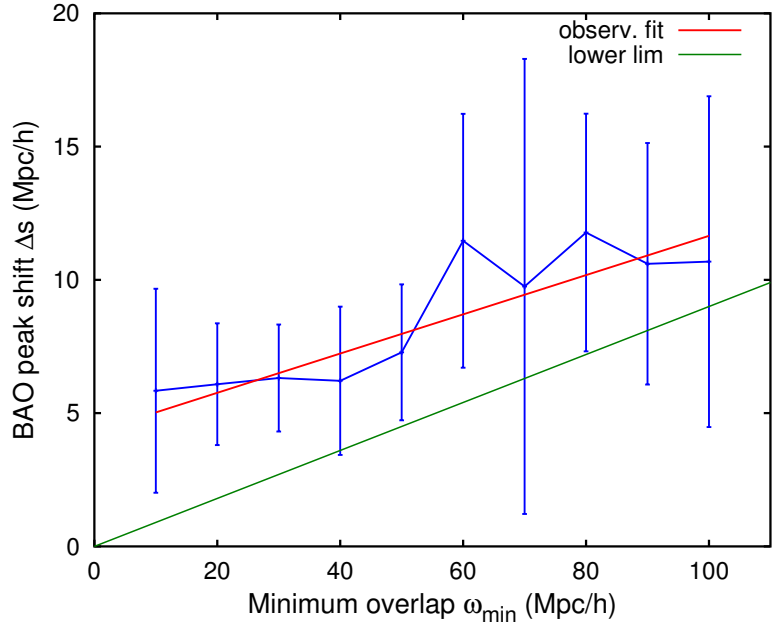

Figure 2. Overlap dependence of the BAO peak shift. BAO peak shift $\Delta s:=s_{\text {non-sc }}-s_{\mathrm{sc}}$, where $s_{\mathrm{sc}}$ and $s_{\text {non-sc }}$ are the median estimates of the centres of the best-fit Gaussians to $\xi-\xi_{3}$ for LRG pairs that overlap superclusters (sc) and those that do not (non-sc), respectively. The error bars show a robust estimate of the standard deviation, $\sigma(\Delta s)$, defined here as 1.4826 times the median absolute deviation of $\Delta s$. At each $\omega_{\min }$, these statistics are calculated over 32 bootstrap resamplings of the observational data. Four out of the 320 Gaussian fits in the supercluster-overlap case failed and were ignored in calculating these statistics; no failures occurred for the 320 non-supercluster-overlap cases. A linear least-squares best fit relation $\Delta s=4.3 h^{-1} \mathrm{Mpc}+0.07 \omega_{\min }$ is shown with a red line. The most significant individual rejection of a zero shift is $\Delta s=(6.3 \pm 2.0) h^{-1} \mathrm{Mpc}$ for $\omega_{\min }=30 h^{-1} \mathrm{Mpc}$, a $3.1 \sigma$ (Gaussian) rejection. Since bootstraps are used, this estimate is conservative: $\sigma(\Delta s)$ is expected to be an overestimate of the true uncertainty (Fisher et al. 1994, Sect 2.2). A 9\% shift [Eq. 11] would give $\Delta s=0.09 \omega$. Since $\omega \geq \omega_{\min }$, a scalar-averaging lower expected limit $\Delta s>0.09 \omega_{\min }$ is shown as a green line.

An environment-dependent effect has recently been detected as a six percent compression of the BAO peak location for spatial paths that touch or overlap superclusters of luminous red galaxies (LRGs) in the Sloan Digital Sky Survey (Roukema et al. 2015; SDSS; environment dependence of $\xi$ at smaller scales has also been detected in the SDSS, Chiang et al. 2015).

In this Letter, we check whether the compression is dependent on the minimum overlap between spatial paths and superclusters, as it should be if the effect is induced by the statistically overdense nature of the superclusters.

\section{METHOD}

We modify the previous method (Roukema et al. 2015, Sect 2) in order to allow stronger overlaps. As in the original method, we calculate the correlation function $\xi$ of the "bright" sample of LRGs in the SDSS Data Release 7 (DR7) for pairs of LRGs selected for overlap (Roukema et al. 2015, Sect. 2.3, Fig. 1) (or non-overlap) of superclusters in the survey (Nadathur \& Hotchkiss 2014), using the Landy \& Szalay estimator (Landy \& Szalay 1993) on real and "random" (artificial) catalogues (Kazin et al. 2010). Comoving separations $s$ are calculated assuming the standard $\Lambda$ CDM model (Spergel et al. 2003; Ade et al. 2014) with matter density parameter $\Omega_{\mathrm{m} 0}=0.32$ and dark energy parameter $\Omega_{\Lambda 0}=0.68$. A best-fit cubic $\xi_{3}(s)$ over separations $s \leq 70 h^{-1} \mathrm{Mpc}$ and $s \geq 140 h^{-1} \mathrm{Mpc}$ (i.e. excluding the peak), is found for the tangential signal (pairs 
$\leq 45^{\circ}$ from the sky plane). The procedure is repeated, bootstrap resampling the supercluster catalogue (Nadathur \& Hotchkiss 2014) and the "random" LRG catalogue, several times. The BAO peak location is estimated from the medians and median absolute deviations of the centres of the best-fit Gaussians to $\xi(s)-\xi_{3}(s)$. In this work, minimum overlaps $\omega_{\min }$ in the range $10 h^{-1} \mathrm{Mpc}$ $\leq \omega_{\min } \leq 100 h^{-1} \mathrm{Mpc}$ rather than $\omega_{\min }=1 h^{-1} \mathrm{Mpc}$ are considered. In order that $\xi$ be defined for $s \leq \omega_{\min }$ for these high values of $\omega_{\min }$, we consider a pair of LRGs joined by a comoving spatial path entirely contained within a supercluster to satisfy the overlap criterion.

\section{RESULTS}

Figure 1 shows that for a minimum overlap $\omega_{\min }=60 h^{-1} \mathrm{Mpc}$, the BAO peak is shifted to lower separations $s$ (panel a) than for the complementary set of LRG pairs (panel b). The shift is clearer than for the earlier analysis, which had $\omega_{\min }=1 h^{-1} \mathrm{Mpc}$ (Roukema et al. 2015, Fig. 8). Requiring a stronger overlap yields a stronger shift.

Figure 2 shows the dependence of the shift $\Delta s$ on $\omega_{\min }$. The BAO peak shift for supercluster-overlapping LRG pairs appears to increase from $\Delta s \approx 6-7 h^{-1} \mathrm{Mpc}$ for $\omega_{\min } \approx 50 h^{-1} \mathrm{Mpc}$ to $\Delta s \approx 11 h^{-1} \mathrm{Mpc}$ for greater overlaps. The Pearson product-moment correlation coefficient of $\Delta s$ and $\omega_{\min }$ is 0.87 , with a probability of $P \approx 0.0008$, i.e. a positive correlation is detected to high significance. Thus, the environment dependence of the BAO peak shift is confirmed. Unfortunately, the uncertainties shown in Fig. 2] are too high to infer the details of this correlation from the present data and analysis. The slope and zeropoint of the linear best fit are $0.073 \pm 0.040$ and $4.3 \pm 2.0 h^{-1} \mathrm{Mpc}$, respectively.

As a rough guide to what is expected from scalar averaging, we can use the $9 \%$ shift estimate from Eq. (1), which would give $\Delta s=0.09 \omega$, where the overlap path lengths are approximated as corresponding to $1 \sigma$ overdense regions on the BAO scale, even though in reality, the overdense regions are superclusters, some smaller and some larger than this scale. Since $\omega \geq \omega_{\min }$, this implies a rough scalar-averaging lower expected limit of $\Delta s>0.09 \omega_{\min }$, shown as a green line in Fig. 2

\section{DISCUSSION}

Since the BAO peak location serves as a major tool for cosmological geometry measurements, it is clear that its environment dependence will need to be observationally calibrated and correctly modelled theoretically. It is possible that the effect could also be interpreted within the standard $\Lambda \mathrm{CDM}$ model, as is the case for many large-scale phenomena. For example, observed supervoids on the $200-300 h^{-1} \mathrm{Mpc}$ scale (Nadathur \& Hotchkiss 2014; Szapudi et al. 2015) can be interpreted within the $\Lambda$ CDM model (Hotchkiss et al. 2015), although their occurrence is expected to be rare (Szapudi et al. 2015). In contrast, the study of SDSS DR7 “dim" (or "bright") LRGs via Minkowski functionals on scales ranging up to the BAO peak scale, within a $500 h^{-1} \mathrm{Mpc}$ (or $700 h^{-1} \mathrm{Mpc}$, respectively) diameter region, shows $3-5.5 \sigma$ (or $0.5-2.5 \sigma$ ) inconsistencies with $\Lambda$ CDM simulations Wiegand, Buchert \& Ostermann 2014, Table 1). Minkowski functionals have more statistical power than lower order statistics that are commonly used in analysis of large-scale structure, such as the two- and three-point correlation functions, the correlation dimension or percolation ("friends-of-friends") analyses. This is because all the $n$-point correlation functions would be needed in order to represent the statistical geometrical information that the Minkowski functionals contain.

A possible avenue to studying the environment-dependent BAO shift within the standard approach would be to carry out a Fourier analysis rather than using the two-point correlation function. This would require the development of a supercluster-overlapdependent Fourier analysis method. Another alternative, to avoid having to determine the position of the peak itself, would be comparison of radial to tangential correlation functions directly. This would require correcting for peculiar velocity effects, which are highly anisotropic with respect to the observer.

Interpreting the environment dependence of the BAO peak location reported in this Letter within the standard $\Lambda$ CDM model would require the comoving length scale at which the Universe is rigid in comoving coordinates to be pushed up to a scale greater than $105 h^{-1} \mathrm{Mpc}$. The environment dependence (e.g. the $\Delta s\left(\omega_{\min }\right)$ relation) would have to be modelled within a rigid comoving background that can only exist at larger scales, at which no sharp statistical feature that can function as a standard ruler is presently known. This leads to a Mach's principle type of concern that at recent epochs, it is difficult to have confidence that the standard comoving coordinate system is correctly attached to an observational extragalactic catalogue (peculiar velocity flow analyses indicate similar concerns, Wiltshire et al. 2013). Interpretation within the scalar averaging approach should be easier because its description of fluctuation properties and the cosmological expansion rate is environment-dependent.

Nevertheless, within the rigid comoving background framework (i.e. the standard model), small shifts in the BAO peak location have been predicted analytically and from $N$-body simulations (Desiacques et al. 2010; Sherwin \& Zaldarriaga 2012), while BAO reconstruction techniques (Padmanabhan \& White 2009; Padmanabhan et al. 2012; Schmittfull et al. 2015) have been developed to attempt to evolve galaxies' positions backwards in cosmological time, using a blend of theoretical calculations and $N$-body models. The expected mean shift in the BAO peak location is less than one percent, i.e. an order of magnitude less than what we find for the shift conditioned on $\omega_{\min } \geq 60 h^{-1} \mathrm{Mpc}$. The amplitude of the shifts found in these calculations is constrained by the assumption that curvature averages out on the assumed background, i.e. that a conservation law for instrinsic curvature holds globally (Buchert \& Carfora 2008).

Theoretical work is underway in the scalar averaging approach, which general-relativistically extends the standard model, allowing the restrictive assumption of a conservation law for intrinsic curvature to be dropped (Buchert \& Carfora 2008). The physical origin of curvature deviations from the background on scales as large as the BAO scale can then be thought of as following from the non-existence of a conservation law for intrinsic curvature. To reconstruct the primordial comoving galaxy positions more accurately than in the standard model, i.e. to allow flexible comoving curvature that varies with the matter density and the extrinsic curvature tensor across a spatial slice, relativistic Lagrangian perturbation theory (Buchert \& Ostermann 2012; Buchert, Nayet \& Wiegand 2013; (Alles et al. 2015) is available for analytically guided calculations. $N$-body simulations in which the growth of inhomogeneities is matched by inhomogeneous metric evolution will most likely also be needed to develop numerical confidence in what could be called "relativistic BAO reconstruction". 


\section{CONCLUSION}

No matter which approach is chosen, analytical, numerical and observational work will be required if the BAO peak location is to correctly function as a standard ruler for cosmological geometrical measurements, since the evidence is strong $(P<0.001)$ that it is strongly affected by structure formation. Moreover, the formation of superclusters - in reality, filamentary and spiderlike distributions of galaxies (Einasto et al.2014) rather than the spherically symmetric objects assumed here for calculational speed-can now be tied directly to a sharp statistical feature of the primordial pattern of density perturbations.

\section{ACKNOWLEDGMENTS}

Thank you to Mitsuru Kokubo for useful comments. The work of T.B. was conducted within the "Lyon Institute of Origins" under grant ANR-10-LABX-66. T.B. acknowledges financial support from CONICYT Anillo Project (ACT-1122) and UMIFCA (Laboratoire Franco-Chilien d'Astronomie, UMI 3386, CNRS/INSU, France, and Universidad de Chile) during a lecturing visit. A part of this project was funded by the National Science Centre, Poland, under grant 2014/13/B/ST9/00845. J.J.O. acknowledges support for part of this work from a National Science Centre, Poland, Etiuda 2 grant. Part of this work consists of research conducted within the scope of the HECOLS International Associated Laboratory, supported in part by the Polish NCN grant DEC-2013/08/M/ST9/00664. A part of this project has made use of computations made under grant 197 of the Poznań Supercomputing and Networking Center (PSNC). Funding for the SDSS and SDSS-II has been provided by the Alfred P. Sloan Foundation, the Participating Institutions, the National Science Foundation, the U.S. Department of Energy, the National Aeronautics and Space Administration, the Japanese Monbukagakusho, the Max Planck Society, and the Higher Education Funding Council for England. The SDSS Web Site is http://www.sdss.org We gratefully acknowledge use of the Kazin et al. (2010) version of SDSS DR7 real and random galaxies at http://cosmo.nyu.edu/ eak306/SDSS-LRG.html and of v11.11.13 of the Nadathur \& Hotchkiss (2014) supercluster catalogue at http://research.hip.fi/user/ nadathur/download/dr7catalogue

\section{REFERENCES}

Ade, P. A. R., Aghanim, N., Armitage-Caplan, C., et al. 2014, A\&A, 571, A16, [arXiv: 1303.5076]

Alles, A., Buchert, T., Roumi, F. A., \& Wiegand, A. 2015, PRD, 92, 023512, [arXiv: 1503.02566]

Buchert, T. 2001, Gen. Rel. Grav., 33, 1381, [arXiv:gr-qc/0102049]

Buchert, T. 2008, Gen. Rel. Grav., 40, 467, [arXiv:0707.2153]

Buchert, T. \& Carfora, M. 2008, ClassQuantGra, 25, 195001, [arXiv:0803.1401]

Buchert, T., Nayet, C., \& Wiegand, A. 2013, PRD, 87, 123503, [arXiv: 1303.6193]

Buchert, T. \& Ostermann, M. 2012, PRD, 86, 023520, [arXiv: 1203.6263]

Chiang, C.-T., Wagner, C., Sánchez, A. G., Schmidt, F., \& Komatsu, E. 2015, ArXiv e-prints, [arXiv: 1504.03322]

Cole, S., Percival, W. J., Peacock, J. A., et al. 2005, MNRAS, 362, 505, [arXiv:astro-ph/0501174] de Jong, R. S., Bellido-Tirado, O., Chiappini, C., et al. 2012, in McLean I. S., Ramsay S. K., Takami H., eds, Ground-based and Airborne Instrumentation for Astronomy IV Vol. 8446 of Society of PhotoOptical Instrumentation Engineers (SPIE) Conference Series. p. 84460T, [arXiv: 1206.6885]

Desjacques, V., Crocce, M., Scoccimarro, R., \& Sheth, R. K. 2010, PRD, 82, 103529, [arXiv: 1009.3449]

Einasto, J., Einasto, M., Gottlöber, S., et al. 1997, Nat., 385, 139, [arXiv:astro-ph/9701018]

Einasto, M., Lietzen, H., Tempel, E., et al. 2014, A\&A, 562, A87, [arXiv: 1401.3226]

Eisenstein, D. J. \& Hu, W. 1998, ApJ, 496, 605, [arXiv:astro-ph/9709112]

Eisenstein, D. J., Zehavi, I., Hogg, D. W., et al. 2005, ApJ, 633, 560, [arXiv:astro-ph/0501171]

Ellis, G. F. R. \& Stoeger, W. 1987, ClassQuantGra, 4, 1697

Fisher, K. B., Davis, M., Strauss, M. A., Yahil, A., \& Huchra, J. 1994, MNRAS, 266, 50, [arXiv:astro-ph/9307001]

Hotchkiss, S., Nadathur, S., Gottlöber, S., et al. 2015, MNRAS, 446, 1321, [arXiv: 1405.3552]

Kazin, E. A., Blanton, M. R., Scoccimarro, R., et al. 2010, ApJ, 710, 1444, [arXiv:0908.2598]

Landy, S. D. \& Szalay, A. S. 1993, ApJ, 412, 64

Lemaître, G. 1927, Annales de la Société Scientifique de Bruxelles, 47, 49

Levi, M., Bebek, C., Beers, T., et al. 2013, ArXiv e-prints, [arXiv: 1308.0847]

Nadathur, S. \& Hotchkiss, S. 2014, MNRAS, 440, 1248, [arXiv: 1310.2791]

Padmanabhan, N. \& White, M. 2009, PRD, 80, 063508, [arXiv:0906.1198]

Padmanabhan, N., Xu, X., Eisenstein, D. J., et al. 2012, MNRAS, 427, 2132, [arXiv: 1202.0090]

Refregier, A., Amara, A., Kitching, T. D., et al. 2010, ArXiv e-prints, [arXiv: 1001.0061]

Roukema, B. F., Buchert, T., Ostrowski, J. J., \& France, M. J. 2015, MNRAS, 448, 1660, [arXiv: 1410.1687]

Schmittfull, M., Feng, Y., Beutler, F., Sherwin, B., \& Yat Chu, M. 2015, ArXiv e-prints, [arXiv: 1508.06972]

Sherwin, B. D. \& Zaldarriaga, M. 2012, PRD, 85, 103523, [arXiv: 1202.3998]

Spergel, D. N., Verde, L., Peiris, H. V., et al. 2003, ApJSupp, 148, 175, [arXiv:astro-ph/0302209]

Szapudi, I., Kovács, A., Granett, B. R., et al. 2015, MNRAS, 450, 288, [arXiv: 1405.1566]

Tyson, J. A., Wittman, D. M., Hennawi, J. F., \& Spergel, D. N. 2003, Nuclear Physics B Proceedings Supplements, 124, 21, [arXiv:astro-ph/0209632]

Wiegand, A., Buchert, T., \& Ostermann, M. 2014, MNRAS, 443, 241, [arXiv: 1311.3661]

Wiltshire, D. L., Smale, P. R., Mattsson, T., \& Watkins, R. 2013, PRD, 88, 083529, [arXiv: 1201.5371] 